\title{
Sleeve-type Torque Sensor Based on Effect of Strain Induction Applied to Wide-blade Drill Rod
}

\author{
Dongmin Li, ${ }^{1,2,3 *}$ Yuanzhi Zhao, ${ }^{1}$ Shiming Zhu, ${ }^{1}$ Hengxuan Luan, ${ }^{1}$ and Qiankun $\mathrm{Ma}^{4}$ \\ ${ }^{1}$ Department of Mechanical and Electronic Engineering, Shandong University of Science and Technology, \\ 223 Daizong Street, Tai'an, Shandong Province 271019, P.R. China \\ ${ }^{2}$ State Key Laboratory of Fluid Power and Mechatronic System, Zhejiang University, \\ 866 Yuhangtang Road, Hangzhou, Zhejiang Province 310058, P.R. China \\ ${ }^{3}$ State Key Laboratory of Mining Disaster Prevention and Control Co-founded by Shandong Province and the \\ Ministry of Science and Technology, Shandong University of Science and Technology, \\ 579 Qianwangang Road, Qingdao, Shandong Province 266590, P.R. China \\ ${ }^{4}$ Beijing Aviation TaiLong Technoloy Co., Ltd., \\ 54 Songzhuang Town Economic Development Zone, Beijing 101149, P.R. China
}

(Received July 27, 2019; accepted December 2, 2019)

Keywords: sleeve-type torque sensor, drill rod, linearity, coal mine, effect of strain induction

To obtain the dynamic torques exerted at different location points on a wide-blade drill rod, a sleeve-type torque sensor is proposed and designed. Firstly, the relationship model between torque and voltage is built on the basis of a mechanical theory. The sensor is designed on the basis of the effect of strain induction, and its structure is designed according to the structural type of the wide-blade drill rod. Furthermore, the deformation of strain gauges is determined by finite element analysis, and the relationship between strain and torque is established. Secondly, the modal analysis of the torque sensor is performed. The hardware circuit for detecting torque is designed. Finally, the performance test is performed and, according to the test results, the performance of the sensor meets the requirements of actual working conditions. Therefore, torque can be measured using a torque sensor for the drill rod, which helps solve the problem of drill rod sticking in underground coal mine.

\section{Introduction}

Drill rod sticking is a fatal issue during a drilling operation in underground coal mine, ${ }^{(1,2)}$ which is shown in Fig. 1; thus, some parameters, e.g., torque, rotation speed, and feeding force, are investigated to clarify the essence of drill rod sticking. A strain-gauge-type force sensor puts forward the mathematical relationship model between force and pressure, and the experimental results show that the maximum calculation difference can be reduced. ${ }^{(3)}$ The static-active design theory of a piezoelectric six-axis force sensor is established and static characteristic parameters are tested using a numerical simulation model with a modeling approach and a load application method, respectively. The measured results are in agreement with the analytical results from the static-active design method. ${ }^{(4)}$

*Corresponding author: e-mail:1dm753@163.com

https://doi.org/10.18494/SAM.2020.2533 


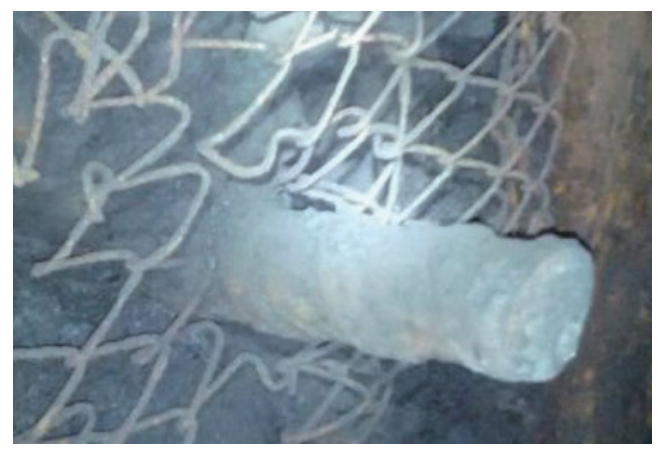

Fig. 1. (Color online) Actual scene of drill rod sticking in underground coal mine.

However, current torque sensors are not applicable to a wide-blade drill rod; thus, a novel one is designed and studied. Moreover, the sections are arranged as follows: (1) the sensor's structure is designed; (2) the theoretical relationships among strain, torque, and voltage are deduced; (3) the finite element analysis of the sensor structure is performed; (4) the hardware circuit for converting strain into voltage is designed; (5) the sensor performance is tested.

\section{Torque Detection Methodology}

A torque sensor works under the effect of strain induction, ${ }^{(5-7)}$ i.e., when torque is exerted on the sensor, the sensor generates the corresponding strain, then the strain is converted into the corresponding resistance, which is regarded as the effect of strain induction. The structure of the sleeve-type torque sensor is designed as follows: Firstly, the internal surface of the rod sleeve is coupled with the external surface of the wide-blade drill rod to smoothen the surface of the drill rod. Furthermore, the strain gauge is pasted on the external surface of the rod sleeve. Therefore, the detection of torque with the torque sensor is performed as follows: When the torque is exerted on the drill rod, the strain gauge generates the corresponding strain. Then, the strain is converted into the corresponding resistance due to the effect of strain induction. Next, the resistance is converted into voltage by the bridge circuit. Finally, a formula of the relationship between strain and output voltage is obtained.

As we all know, resistance is expressed as Eq. (1), where $R, \rho, l$, and $A$ denote the resistance, resistivity, length, and cross-sectional area of the wire, respectively.

$$
R=\frac{\rho \cdot l}{A}
$$

According to the theory of material mechanics and the total differential equation, the strain sensitivity of the wire can be represented by Eq. (2); thus, the strain can be converted into the resistance using Eq. (3), where $\Delta R, K, \mu, \lambda, E$, and $\varepsilon_{l}$ denote the resistance increment, strain sensitivity, Poisson's ratio, piezoresistive coefficient, elasticity modulus, and axial strain of the wire, ${ }^{(8,9)}$ respectively. 


$$
\begin{gathered}
K=\frac{d R / R}{d l / l}=\frac{\Delta R / R}{\varepsilon_{l}}=1+2 \mu+\lambda E \\
\frac{\Delta R}{R}=(1+2 \mu+\lambda E) \cdot \varepsilon_{l}=K \cdot \varepsilon_{l}
\end{gathered}
$$

Furthermore, the voltage is output by a full electrical bridge circuit composed of four strain gauges; thus, the resistance is converted into the voltage using Eq. (4), where $U_{o}$ and $U_{i}$ denote the output and input voltages, respectively.

$$
\frac{U_{o}}{U_{i}}=\frac{\Delta R}{R}=K \cdot \varepsilon_{l}
$$

When the drill rod is hollow, the relationship between the strain and torque of the drill rod is represented by Eq. (5) according to the theory of material mechanics, ${ }^{(10)}$ where $D, d$, and $T$ denote the external diameter, internal diameter, and torque of the drill rod, respectively.

$$
\varepsilon_{l}=\frac{16 D(1+\mu)}{\pi \cdot E\left(D^{2}+d^{2}\right)} T
$$

Finally, from the above deduction, the relationship between the torque of the drill rod and the voltage converted can be represented by Eq. (6), i.e., the torque of the drill rod is converted into the output voltage.

$$
T=\frac{\pi \cdot E\left(D^{2}+d^{2}\right)}{16 D(1+\mu)} \cdot \frac{U_{o}}{U_{i} \cdot K}
$$

\section{Finite Element Analysis of the Torque Sensor}

\subsection{Model of the torque sensor}

The sleeve-type torque sensor is designed and shown in Fig. 2 according to the profile and dimensions of the wide-blade drill rod. It consists of an upper rod sleeve, a lower rod sleeve, a strain gauge, a battery, an electric brush, and a signal output circle. Among these components, the upper rod sleeve (indexed as "6") and lower rod sleeve (indexed as "7") are connected together by bolts, and the drill rod (indexed as "1") passes through their center hole.

Furthermore, the internal screw thread of the rod sleeves matches with the external screw thread of the drill rod; thus, the two rod sleeves move with the drill rod synchronously under friction. The electric bridge consisting of four strain gauges (indexed as " 5 ") adhered to the rod sleeves is established to deliver the voltage signal to the electric brush (indexed as " 3 ") via a signal output circle (indexed as "2"). The two signal output circles do not touch each other, and 


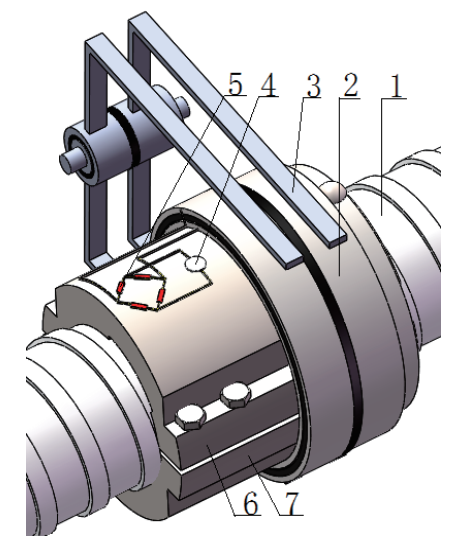

Fig. 2. (Color online) Three-dimensional model of the sleeve-type torque sensor. 1-drill rod, 2-signal output circle, 3-electric brush, 4-battery, 5-strain gauge, 6-upper rod sleeve, and 7-lower rod sleeve.

they are insulated from the rod sleeves by the insulation sandwich; in addition, two of the strain gauges adhere in the $45^{\circ}$ direction from the axial direction of the drill rod, and correspondingly, the other two strain gauges adhere in the $135^{\circ}$ direction. Furthermore, the electric bridge is powered by a button battery (indexed as “4").

When the drill rod rotates, the rod sleeves and signal output circles rotate simultaneously, and the torque signal generated from the drill rod is converted into the strain, resistance, and voltage signals successively, and finally delivered to the controller via the two electric brushes; therefore, the torque is obtained and expressed theoretically as Eq. (6).

\subsection{Finite element analysis of the sensor}

The model of the torque sensor is simplified to increase the analysis efficiency without affecting the analysis quality. In contrast to the model shown in Fig. 2, the simplified model has the following features: (1) The drill rod is shortened and is as long as the torque sensor. (2) The screw threads are omitted; however, the simulated preload still acts on two rod sleeves. (3) The signal output circle and electric brush are not involved. Finally, the simplified model is shown in Fig. 3.

Next, the parameters of the torque sensor and drill rod can be set as shown in Table 1 . Among the parameters, $\mathrm{VoS}$ is the parameter of the torque sensor and VoR is the parameter of the drill rod. Furthermore, the contact type between the torque sensor and the drill rod is set to "friction", and that between the torque sensor and the strain gauges is set to "binding". As for mesh generation, ${ }^{(11,12)}$ a tetrahedral mesh is adopted, and the mesh on the friction surface is generated densely to improve the analysis accuracy.

Next, the constraints are set. The stress is exerted on four contact surfaces of the bolts and rod sleeves in order to simulate the pretightening force, e.g., $10 \mathrm{MPa}$. One end of the drill rod is fixed in order to simulate the loads exerted on the drill rod; moreover, the driving torque is exerted on the other end of the drill rod and gradually loaded from 300 to $3200 \mathrm{~N} \cdot \mathrm{m}$ with 12 steps. Finally, the simulation result is shown in Fig. 4. It indicates that the sensor 


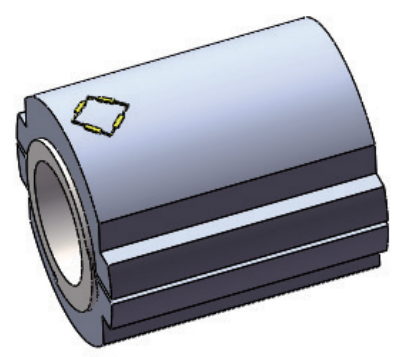

Fig. 3. (Color online) Simplified model of the torque sensor.

Table 1

Parameters of the torque sensor and drill rod.

\begin{tabular}{lccccc}
\hline Item & Elasticity modulus $(\mathrm{MPa})$ & Material & Poisson's ratio & Yield strength $(\mathrm{MPa})$ & Density $\left(\mathrm{kg} / \mathrm{m}^{3}\right)$ \\
\hline VoS & $7.1 \times 10^{4}$ & Aluminium alloy & 0.33 & $280 \times 10^{3}$ & 27.7 \\
VoR & $2 \times 10^{5}$ & Steel & 0.3 & 250 & $7.85 \times 10^{3}$ \\
\hline
\end{tabular}
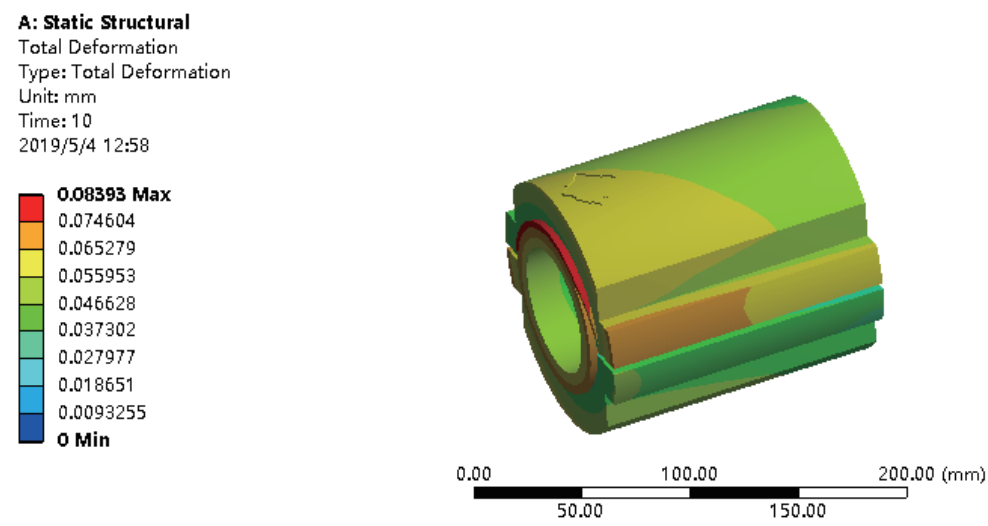

Fig. 4. (Color online) Cloud map of strain of the torque sensor.

induces a deformation of about $0.055953-0.065279 \mathrm{~mm}$ under the torque of $3200 \mathrm{~N} \cdot \mathrm{m}$, and the mean deformation is $0.060616 \mathrm{~mm}$. Similarly, the results generated from the other 11 steps are listed with coordinates successively as follows: $(150 \mathrm{~N} \cdot \mathrm{m}, 0.0040603 \mathrm{~mm}),(300 \mathrm{~N} \cdot \mathrm{m}$, $0.00624945 \mathrm{~mm}),(600 \mathrm{~N} \cdot \mathrm{m}, 0.0106568 \mathrm{~mm}),(900 \mathrm{~N} \cdot \mathrm{m}, 0.01633 \mathrm{~mm}),(1200 \mathrm{~N} \cdot \mathrm{m}, 0.0232635$ $\mathrm{mm}),(1500 \mathrm{~N} \cdot \mathrm{m}, 0.0268415 \mathrm{~mm}),(1800 \mathrm{~N} \cdot \mathrm{m}, 0.032977 \mathrm{~mm}),(2100 \mathrm{~N} \cdot \mathrm{m}, 0.0387995 \mathrm{~mm}),(2400$ $\mathrm{N} \cdot \mathrm{m}, 0.044724 \mathrm{~mm}),(2700 \mathrm{~N} \cdot \mathrm{m}, 0.050675 \mathrm{~mm})$, and $(3000 \mathrm{~N} \cdot \mathrm{m}, 0.0566355 \mathrm{~mm})$.

Furthermore, a fitted function relationship between the torque exerted and the deformation generated with the least squares method is obtained as Eq. (7) on the basis of the above 12 groups of data; among them, $\mathrm{m}_{1}$ and $\mathrm{m}_{2}$ are both constants, i.e., 18.6861 and 0.0699 are set on the basis of the simulation results and Eq. (2), respectively.

$$
l=m_{1} \cdot T+m_{2}
$$

Here, $l$ is deformation $(\mu \mathrm{m})$ and $T$ is torque $(\mathrm{kN} \cdot \mathrm{m})$. 


\subsection{Modal analysis of the sensor}

To investigate the vibration of the torque sensor during operation, a modal analysis of the sensor is performed after the driving torque and constrains are exerted on the sensor, and the front 6-phase vibration modes are obtained and shown in Figs. 5-10. The six-phase vibration frequencies are 3257.6, 3614.6, 5532.5, 6527.5, 6880.5, and $7097 \mathrm{~Hz}$, respectively.
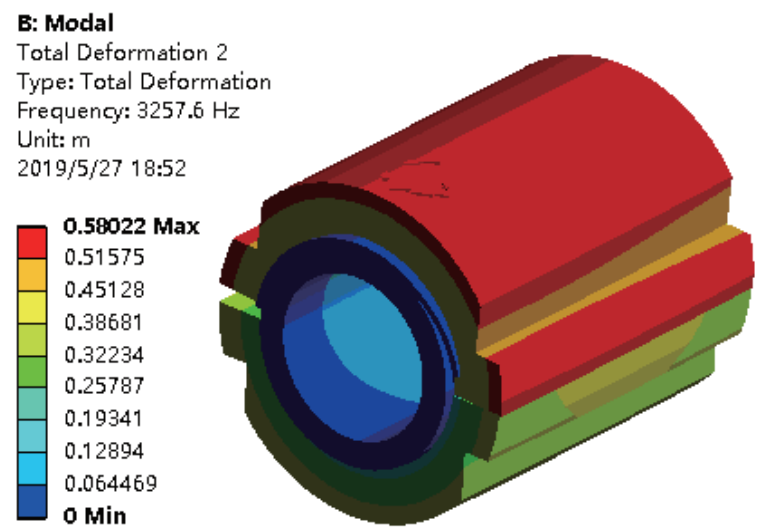

Fig. 5. (Color online) One-phase modal mode.

B: Modal

Total Deformation 4

Type: Total Deformation Frequency: $5532.5 \mathrm{~Hz}$ Unit: $m$ 2019/5/27 18:56
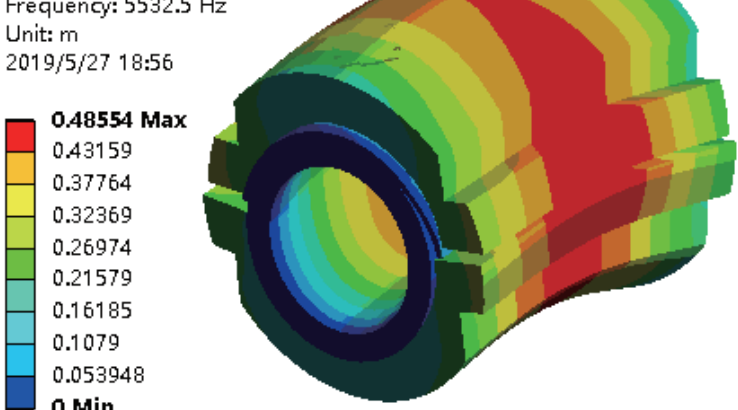

Fig. 7. (Color online) Three-phase modal mode.
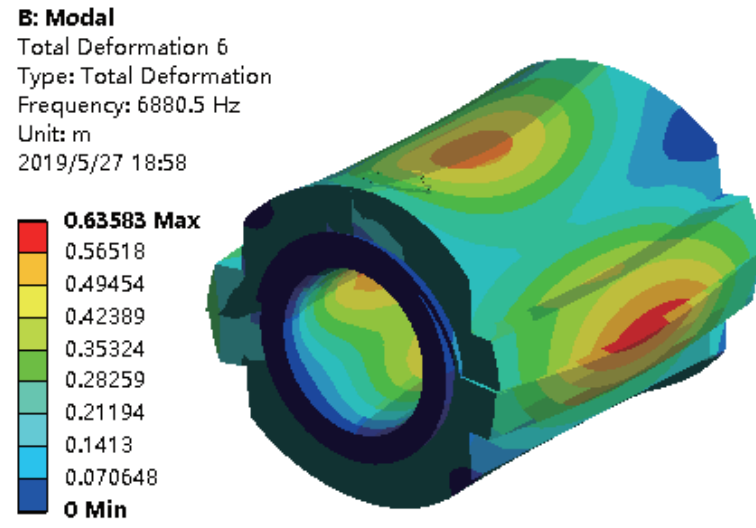

Fig. 9. (Color online) Five-phase modal mode.

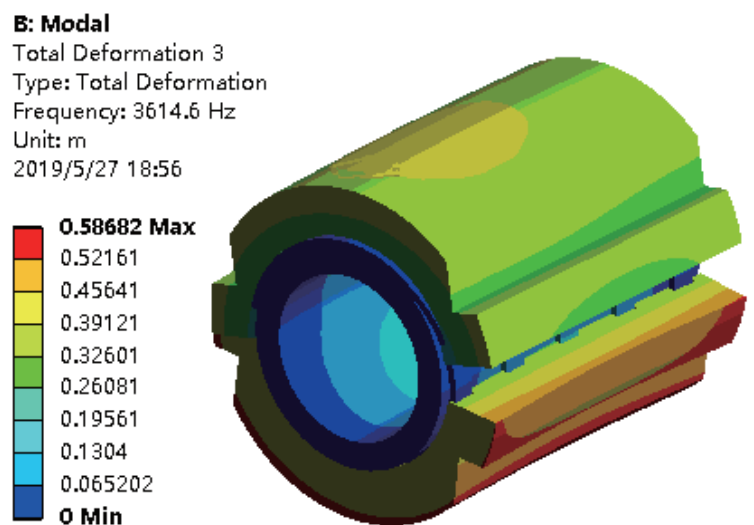

Fig. 6. (Color online) Two-phase modal mode.

\section{B: Modal}

Total Deformation 5

Type: Total Deformation

Frequency: $6527.5 \mathrm{~Hz}$

Unit: $\mathrm{m}$

2019/5/27 18:58

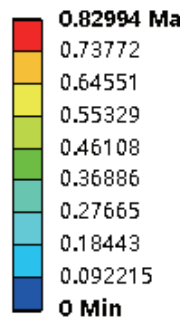

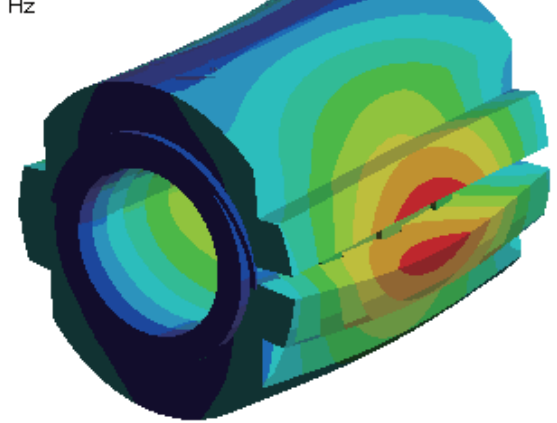

Fig. 8. (Color online) Four-phase modal mode.

\section{B: Modal}

Total Deformation 7

Type: Total Deformation

Frequency: 7097. Hz

Unit: $m$

2019/5/27 18:59
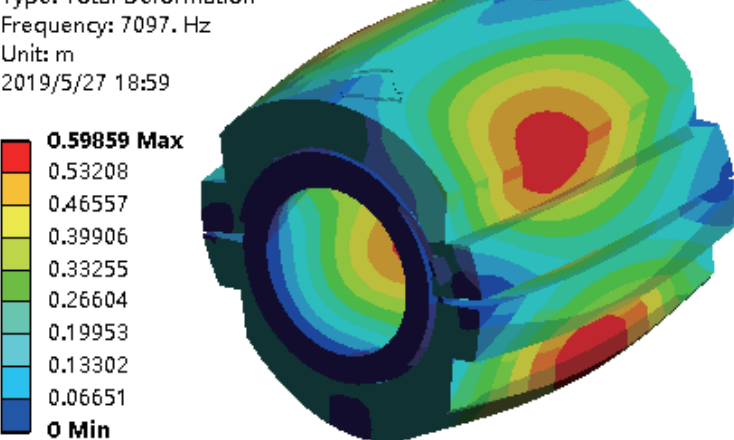

Fig. 10. (Color online) Six-phase modal mode. 
For the torque sensor to rotate at a relatively low speed, the relationship between the rotation speed and the frequency is determined and shown as

$$
n=60 \cdot f \text {. }
$$

At the given one-phase modal natural frequency of $3257.6 \mathrm{~Hz}$, the corresponding critical rotation speed should be $195456 \mathrm{r} / \mathrm{min}$ according to Eq. (8); however, the maximum rotation speed of the torque sensor is only $500 \mathrm{r} / \mathrm{min}$ according to the actual working conditions; therefore, no resonance will occur.

\section{Design of Hardware Circuit}

The torque exerted on the drill rod is converted into the corresponding voltage and finally displayed on the displayer via the hardware circuit; therefore, the hardware circuit is designed and shown in Fig. 11.

The hardware circuit consists of voltage-stabilizing circuit, strain gauge circuit, simplifying circuit, filter circuit, ${ }^{(13,14)}$ A/D conversion circuit, single-chip microcomputer circuit, and display circuit. When it works, the power is supplied by the voltage-stabilizing circuit. Then, a full-bridge circuit composed of four strain gauges converts the torque into the strain, resistance, and voltage successively, and the last voltage signal is transferred to the amplifying circuit in the end. Next, the signal amplified is transferred to the single-chip microcomputer via resistancecapacitance filtering and A/D conversion. Finally, the torque is transferred to the display circuit and displayed after the signal is processed by the single-chip microcomputer. ${ }^{(15-17)}$

\section{Performance Test for the Torque Sensor}

The experimental device consists of the torque sensor, detection instrument, loads, drill rod, and frame. The entire device and detection section are shown in Figs. 12(a) and 12(b), respectively.

When the test experiment starts, the drill rod rotates under the driving torque. The load is exerted on the drill rod; therefore, the torque is generated on the entire drill rod. Furthermore, the torque sensor detects the torque and transfers it to the detection instrument.

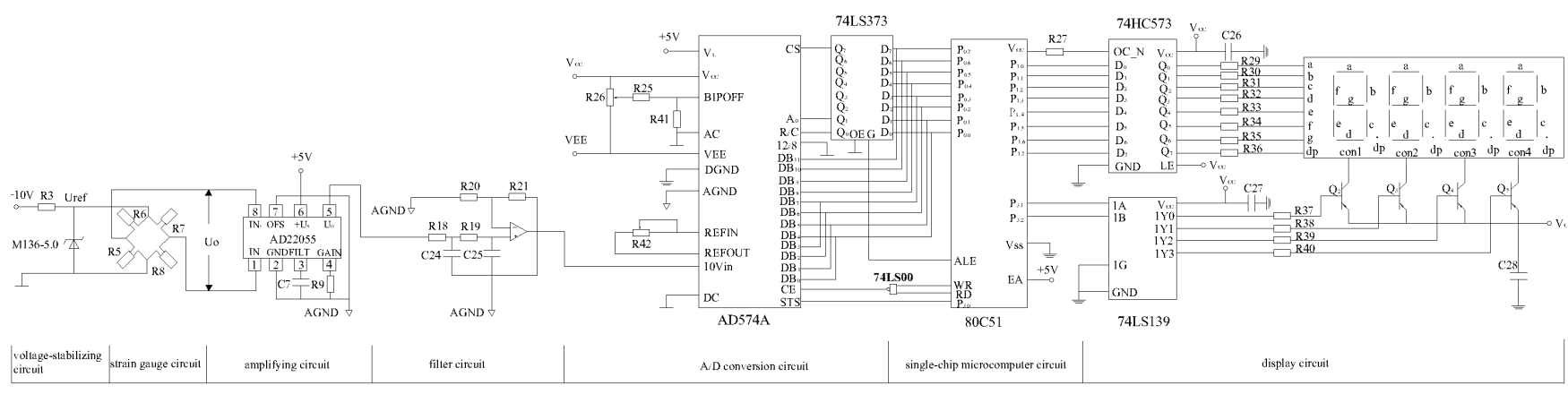

Fig. 11. Hardware circuit on torque conversion. 


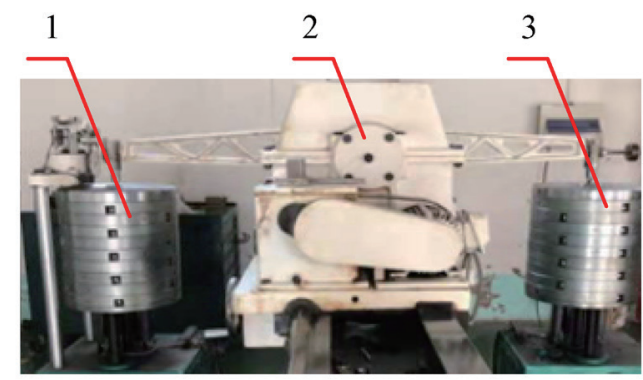

(a)

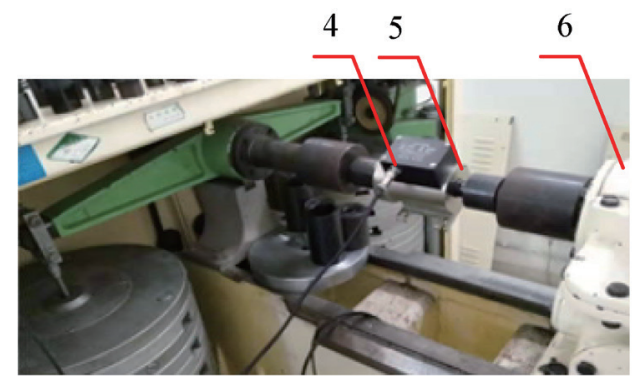

(b)

Fig. 12. (Color online) Experimental device. (a) 1-positive load, 2-frame, and 3-reverse load. (b) 4-detection instrument, 5-torque sensor, and 6-driving torque.

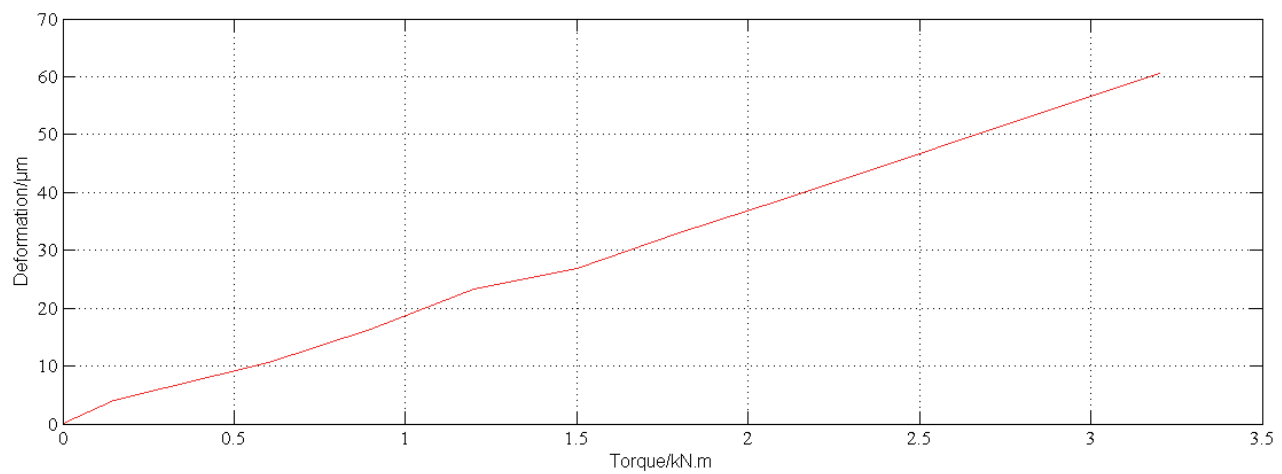

Fig. 13. (Color online) Fitted relationship curve between torque and deformation.

The first driving torque given is $300 \mathrm{~N} \cdot \mathrm{m}$ and is subsequently increased to $300 \mathrm{~N} \cdot \mathrm{m}$ at every step, so the driving torque at the 10 th step is $3000 \mathrm{~N} \cdot \mathrm{m}$, and the last torque is $3200 \mathrm{~N} \cdot \mathrm{m}$ obviously. The experiments are performed 10 times repeatedly under the same experimental conditions in the laboratory and then the mean value for every level torque is obtained to improve the test accuracy; thus, 11 mean torques for the corresponding strain are obtained. Therefore, a fitted line is obtained on the basis of the above mean torques and shown in Fig. 13. It indicates that the relationship between torque and deformation is almost linear, and the linearity is calculated as

$$
\gamma_{L}=\frac{\Delta l}{y_{F S}} \times 100 \%
$$

where $\gamma_{L}$ is the linearity (nondimensional quantity), $\Delta l$ is the maximum nonlinear error $(\mu \mathrm{m})$, and $y_{F S}$ is the full-scale value $(\mu \mathrm{m})$.

When the full-scale value is $60 \mu \mathrm{m}$, the linearity reaches $1.7 \%$. In addition, the sensitivity is about 18 , and the hysteresis and repeatability are both sound according to the experimental process. Therefore, the results prove that the performance of the torque sensor meets the application requirements. 


\section{Conclusions}

To detect the torque exerted on a drill rod dynamically, a sleeve-type torque sensor is designed. As a result, the following conclusions are made by theoretical derivation, mechanical analysis, hardware design, and performance test:

Firstly, the relationship model between torque and voltage is built by theoretical derivation, and determined to be linear.

Moreover, the relationship between torque and deformation is built by mechanical analysis, and determined to be also linear. The modal analysis of the torque sensor shows that no resonance occurs, so the structure of the torque sensor is considered feasible.

Next, the hardware circuit for signal conversion is designed. The voltage is processed and transferred to the displayer by the hardware circuit after the torque is converted into voltage by the strain gauge bridge circuit.

Finally, the performance of the torque sensor is tested with the experimental device. The test results show that the linearity, sensitivity, hysteresis, and repeatability all meet the application requirements; therefore, the sleeve-type torque sensor is considered feasible.

\section{Acknowledgments}

This work was supported by the Open Fund from the State Key Laboratory of Mine Disaster Prevention and Control co-founded by Shandong Province and the Ministry of Science and Technology, China (Grant No. MDPC201609), the Open Foundation of the State Key Laboratory of Fluid Power Transmission and Mechatronic Systems, China (Grant No. GZKF201614), the Project of the Natural Science Foundation of Shandong Province, China (Grant No. ZR2015EM042), the Project of the Shandong Province Higher Educational Science and Technology Program, China (Grant No. J18KB020), and the Tai'an City Science and Technology Development Program, China (Grant No. 2017GX0033).

\section{References}

1 Y. L. Wang, Y. Sun, Z. F. Wang, and W. B. Song: Meitan Xuebao 40 (2015) 119. https://doi.org/10.13225/j.cnki. jecs.2014.0536 (in Chinese)

2 D. M. Li and D. J. Huang: Int. J. Autom. Technol. 10 (2016) 827. https://doi.org/10.20965/ijat.2016.p0827

3 T. W. Gu, F. Shang, D. R. Kong, and C. D. Xu: Rev. Sci. Instrum. 90 (2019) 055111. https://doi. org/10.1063/1.5083895

4 J. Liu, M. Li, L. Qin, and J. C. Liu: Sensors-Basel 14 (2014) 659. https://doi.org/10.3390/s140100659

5 Q. Wang, H. K. Gao, B. Jiang, J. Yang, and Z. J. Lv: Arab. J. Geosci. 11 (2018) 357. https://doi.org/10.1007/ s12517-018-3715-z

6 A. M. Madni, J. B. Vuong, D. C. H. Yang, and B. Huang: IEEE Sens. J. 7 (2007) 800. https://doi.org/10.1109/ jsen.2007.894134

7 D. Pavkovic, J. Dear, and A. Lisac: Control Eng. Pract. 19 (2011) 836. https://doi.org/10.1016/ j.conengprac.2011.04.012

8 S. Y. Kim, S. H. Park, B. G. Choi, I. H. Kang, S. W. Park, J. W. Shin, J. H. Kim, W. K. Baek, K. T. Lim, Y. J. Kim, J. B. Song, and I. Kang: J. Nanosci. Nanotechnol. 18 (2018) 1782. https://doi.org/10.1166/jnn.2018.14989

9 S. Shams, D. Shin, J. Han, J. Y. Lee, K. Shin, and C.-S. Han: IEEE/RSJ Int. Conf. Intelligent Robots \& Systems (IEEE, 2011) 5127-5132. https://doi.org/10.1109/IROS.2011.6094590 
10 H. Zhao: Chin. J. Sens. Actuators 28 (2015) 5. https://doi.org/10.3969/j.issn.1004-1699.2015.05.008 (in Chinese)

11 H. Zhao: Rev. Sci. Instrum. 86 (2015) 055003. https://doi.org/10.1063/1.4919908

12 K. S. Jiang, Y. Y. Zhou, L. B. Han, L. H. Li, Y. Liu, and S. Hu: IEEE Sens. J. 19 (2019) 6595. https://doi. org/10.1109/JSEN.2019.2911392

13 R. Pandey and S. Singh: Wireless Pers. Commun. 107 (2019) 1251. https://doi.org/10.1007/s11277-019-06334-w

14 T. H. Ma: Int. J. Control Autom. 9 (2016) 93. https://doi.org/10.14257/ijca.2016.9.7.09

15 X. Xie and Z. Long: Int. J. Online Eng. 11 (2015) 8. https://doi.org/10.3991/ijoe.v11i8.4881

16 W. Y. He, S. Y. Zhu, and W. X. Ren: Appl. Math. Model. 66 (2019) 29. https://doi.org/10.1016/j.apm.2018.10.005

17 L. Li, C. Sau, T. Fanni, J. G. Li, T. Viitanen, F. Christophe, F. Palumbo, L. Raffo, H. Huttunen, J. Takala, and S. S. Bhattacharyya: J. Syst. Archit. 93 (2019) 1. https://doi.org/10.1016/j.sysarc.2018.12.010

\begin{abstract}
About the Authors
Dongmin Li received his Ph.D. degree from Shandong University of Science and Technology, China, in 2009. He is currently an associate professor at the Department of Mechanical and Electronic Engineering, Shandong University of Science and Technology, China. His research interests are in mechatronics and robotics. (1dm753@163.com)
\end{abstract}

Yuanzhi Zhao is currently a master candidate at the Department of Mechanical and Electronic Engineering, Shandong University of Science and Technology, China. His research interests are in mechatronics and robotics. (1063362103@qq.com)

Shiming Zhu is currently a master candidate at the Department of Mechanical and Electronic Engineering, Shandong University of Science and Technology, China. His research interests are in mechatronics and robotics. (2275001581@qq.com)

Hengxuan Luan received his Ph.D. degree from Shandong University of Science and Technology, China, in 2017. He is currently a lecturer at the Department of Mechanical and Electronic Engineering, Shandong University of Science and Technology, China. His research interests are in mechatronics and fluid power. (158702392@qq.com)

Qiankun Ma is currently a manager at Beijing Aviation TaiLong Technoloy Co., Ltd., China. His research interests are in mechatronics. (1301387065@qq.com) 